\title{
Evaluation of a new rapid test for detection of Legionella pneumophila serogroup | antigen in urine
}

Gino Ciarrocchi', Marco D'anzeo', Elisabetta Farnocchia', Maria Enrica Cimarelli

I S.O. Laboratorio analisi, Sierologia, A.O. Ospedali Riuniti, Ancona

2 S.O. Broncopneumologia, A.O. Ospedale Civile, Jesi

Key words: Legionellosis, Specificity, Sensitivity, Immunochromatographic test

Valutazione di un nuovo test rapido per la ricerca dell'antigene urinario di Legionella pneumophila sierotipo I

\section{SUMMARY}

For the diagnosis of legionellosis, a new rapid immunochromatographic test (V-tesT CORIS BioConcept) for detection of Legionella pneumophila serogroup I antigen in urine was evaluated in 21 samples of patients with legionellosis and 45 samples from patients with other respiratory infections. Results were compared with those obtained by a commercially reference test (BinaxNOW Legionella - Binax). The overall sensitivity was $\mathbf{9 0 . 4 \%}$ for both tests; concordance was $100 \%$; specificity was $100 \%$ as well. In conclusion, the V-tesT CORIS seems to be a prompt and suitable diagnostic tool in legionellosis diagnosis.

\section{INTRODUZIONE}

La legionellosi o Malattia dei Legionari (ML) è una severa forma di polmonite causata dall'infezione di Legionella pneumophila, clinicamente indistinguibile da altre infezioni polmonari. Il battere viene trasmesso principalmente attraverso aerosol contaminati, da fonti umide, quali torri di raffreddamento, impianti di condizionamento. Legionella pneumophila è responsabile del 2-5\% delle polmoniti acquisite in comunità (CAP) e del 5-20\% di quelle nosocomiali (1).

Il ricovero ospedaliero viene deciso in base alla gravità del quadro clinico, con eventualmente ricorso alle unità di cura intensiva. Dal punto di vista epidemiologico, Legionella pneumophila sierotipo 1 è responsabile in Europa e negli USA del 90-95\% dei casi di ML, mentre in Australia e Nuova Zelanda Legionella longbeachae ha prevalenza epidemiologica $(1,8)$.

La frequente severità dei quadri clinici e le aspecificità sintomatologiche rendono indispensabile una rapida e affidabile definizione diagnostica. Tuttavia, nel caso di legionellosi, la certezza della diagnosi è condizionata da molti fattori, tra i quali emergono il quadro di presentazione dell'infezione, l'appropriatezza della richiesta e la qualità dei test di laboratorio impiegati allo scopo. Il test rapido immunocromatografico (IC) si è oramai affermato in campo clinico per l'elevata sensibilità, la pressoché assoluta specificità e la rapidità di esecuzione (3, 4, 5, 6, 7, 9, 10).

L'impiego del test IC, combinato alla ricerca sierologica di IgM anti- Legionella pneumophila sierotipo 1, rappresenta l'iter analitico più efficace nella diagnosi di $\operatorname{ML}(1,2,3,4)$. Scopo del nostro lavoro è stato quello di valutare l'efficacia diagnostica di un nuovo test con tecnica immunocromatografica, impiegato per la ricerca degli antigeni di Legionella pneumophila sierotipo 1 in campioni di urine non concentrate appartenenti a pazienti con accertata legionellosi.

\section{Presentazione dello studio}

Un totale di 21 campioni di urina di pazienti maschi e femmine adulti con diagnosi accertata di legionellosi, formulata secondo i consueti criteri clinici, furono retrospettivamente esaminati per la ricerca degli antigeni urinari di Legionella pneumophila sierotipo 1 mediante un nuovo test rapido di recente introduzione, basato sulla tecnica IC (Legionella V-TesT - CORIS BioConcept, Belgio - distribuito da Alifax, Italia). I pazienti selezionati mostravano quadri clinici di polmonite di differente severità, con imaging di infiltrati polmonari in prevalenza multipli, insieme ad uno o più dei seguenti segni clinici: tosse non produttiva, artromialgie, vomito, diarrea, confusione mentale, feb- bre, mal di testa, iposodiemia e ipofosfatemia.

I campioni di urina positivi per antigeni di Legionella pneumophila sierotipo 1 erano stati precedentemente esaminati mediante un analogo test rapido IC (BinaxNOW Legionella, Binax - USA) e poi conservati a $-30^{\circ} \mathrm{C}$ fino al momento del confronto. I campioni sono stati riesaminati simultaneamente con i due test e la lettura del risultato è avvenuta dopo 15 minuti, come da istruzione operativa per entrambi i test, e ripetuta dopo 30 e 60 minuti.

Il test CORIS si basa sull'impiego di un dispositivo con una membrana contenente anticorpi mono- e policlonali antiLegionella pneumophila coniugati a particelle di oro colloidale; nella parte superiore e inferiore della membrana sono rispettivamente fissati anticorpi anti-Legionella pneumophila sierotipo 1 e un anticorpo di controllo (controllo di migrazione). Nella parte apicale del device viene aggiunta una quantità precisa di urina non concentrata $(110 \mu \mathrm{l})$, la quale diffonderà per gravità lungo la membrana. Il riscontro di una banda colorata di variabile intensità nella specifica posizione per il campione indica un risultato positivo. Per ritenere valido il test una seconda banda deve essere visibile anche a livello del controllo di migrazione.

Il test BinaxNOW è un test immunocromatografico dove anticorpi di coniglio anti-Legionella sierogruppo 1 e anticorpi anti-specie sono assorbiti in due linee di una membrana di nitrocellulosa (linea del paziente e linea di controllo). Un tampone viene immerso nel campione di urina, rimosso e inserito nel dispositivo del kit. Vengono aggiunte due gocce di reagente (tampone citrato/fosfato con Tween 20) che faranno diffondere gli eventuali antigeni lungo la membrana. Il riscontro di una banda colorata anche di debole intensità nella specifica posizione per il campione indica un risultato positivo. Anche per questo test una seconda banda deve essere visibile a livello della linea di controllo.

I risultati dei 21 campioni di urina furono interpretati visivamente dopo 15 minuti, secondo le indicazioni delle istruzioni operative di entrambi i test; la lettura fu altresì ripetuta anche dopo 30 e 60 minuti. Alla lettura iniziale, il test CORIS mostrò 15 campioni con una banda evidente (campioni positivi), 4 campioni con una banda debole (positivi deboli), 2 senza evidenza di banda (campioni negativi); il test BinaxNOW mostrò 14 campioni con una banda evidente (positivi), 5 con una banda debole (positivi deboli), 2 senza evidenza di banda (negativi). Considerando insieme i risultati positivi e border-line, la concordanza totale tra i due test fu del $100 \%$. Effettuando la lettura dopo 30 e 60 minuti, il test CORIS mostrò 17 positivi, 2 positivi deboli, 2 negativi; viceversa, il test BinaxNOW mantenne gli iden-

\section{Corresponding author: Gino Ciarrocchi}

S.O. Laboratorio analisi, Sierologia, A.O. Ospedali Riuniti, Ancona

Tel. 07I-596425I; Fax 07I-5964638

E-mail: ciarrokki@libero.it 
Tabella I. Sensibilità e Specificità di V-TesT CORIS e test BinaxNOW dopo incubazione di 15, 30, 60 minuti

\begin{tabular}{|c|c|c|c|c|c|}
\hline & TEMPO INCUBAZIONE & Positivi & POSITIVI DEBOLI & SEnSIBILITÀ $(\mathrm{N}=2 \mathrm{I})$ & SPECIFICITÀ $(\mathrm{N}=45)$ \\
\hline \multirow{3}{*}{$\begin{array}{l}\text { V-TestT } \\
\text { CORIS }\end{array}$} & I5 minuti & I5 (7I.4\%) & $4(19.0 \%)$ & $90.4 \%$ & $100 \%$ \\
\hline & 30 minuti & 17 (80.9\%) & $2(9.5 \%)$ & $90.4 \%$ & $100 \%$ \\
\hline & 60 minuti & 17 (80.9\%) & $2(9.5 \%)$ & $90.4 \%$ & $100 \%$ \\
\hline \multirow{3}{*}{$\begin{array}{l}\text { Test } \\
\text { Binax NOW }\end{array}$} & I5 minuti & $14(66.6 \%)$ & $5(23.8 \%)$ & $90.4 \%$ & $100 \%$ \\
\hline & 30 minuti & 14 (66.6\%) & $5(23.8 \%)$ & $90.4 \%$ & $100 \%$ \\
\hline & 60 minuti & $14(66.6 \%)$ & $5(23.8 \%)$ & $90.4 \%$ & $100 \%$ \\
\hline
\end{tabular}

tici risultati della prima lettura La valutazione dei risultati positivi + border-line mostrò una sensibilità del $90.4 \%$ per entrambi i test. I 45 campioni di pazienti con altre infezioni respiratorie risultarono tutti negativi ad entrambi i test, dopo incubazione di 15, 30 e 60 minuti, mostrando una specificità del 100\% (Tabella 1).

\section{CONCLUSIONI}

Nell'esame dei 21 campioni di urina non concentrata e valutando come positività anche la debole evidenza della striscia di reazione, sia il test CORIS che il BinaxNOW mostravano complessivamente analoghi livelli di sensibilità (90.4\%). Le lievi bande di positività furono rilevate in 4 casi nel test CORIS ed in 5 casi con il test BinaxNOW, dopo incubazione di 15 minuti dall'aggiunta dell'urina nel device rispettivo. Il campione discordante risultava ben evidente al CORIS, ma debole al BinaxNOW, dopo 15 minuti. La lettura visiva dei test fu ripetuta anche dopo 30 e 60 minuti. La prima differenziazione dei risultati si è evidenziata proprio dopo incubazione di 30 minuti: due dei quattro campioni inizialmente deboli con il $V$-tesT CORIS divennero francamente positivi e restarono tali anche dopo 60 minuti; viceversa, i cinque campioni deboli al test BinaxNOW restarono immutati anche alle letture successive. I due campioni concordemente negativi appartenevano a pazienti con clinica suggestiva e sierologia negativa nel siero di fase acuta, con sieroconversione di IgM su siero convalescente (dati non mostrati). La ricerca dell'antigene urinario risultò negativa a più riprese al tempo della prima diagnosi.

I 45 campioni di pazienti con altre infezioni respiratorie, di cui dieci con infezione pneumococcica, risultarono tutti concordemente negativi, ai tre intervalli di lettura, con entrambi i test, confermando con ciò l'assoluta specificità del test di riferimento e del $V$-tesT CORIS da valutare. L'eccellente performance di quest'ultimo test, perfino migliore in termini di lettura visiva rispetto al BinaxNOW, può risiedere a nostro avviso nella qualità immunologica, ma soprattutto nella ben standardizzata ed oltremodo semplificata procedura, con immissione di una quantità precisa di campione e diffusione per gravità, senza aggiunta di altri liquidi eluenti o reidratanti. In conclusione il $V$-tesT CORIS si è dimostrato, seppure in via preliminare, un presidio diagnositico rapido e affidabile, connotato da eccellenti livelli di sensibilità e specificità.

\section{BIBLIOGRAFIA}

1. Den Boer JW, Yzerman FPF. Diagnosis of Legionella infection in Legionnaires' disease. Eur J Clin Microbiol Infect Dis, 2004; 23: 871-8.

2. Diederen BMW, Kluytmans JAJW, Peeters M. Evaluation of Vircel enzyme-linked immunosorbent assay and indirect immunofluorescence assay for detection of antibodies against Legionella pneumophila. Clin Vaccine Immunol 2006; 13(3): 361-4.

3. Dirven K, Ieven M, Peeters MF, et al. Comparison of three Legionella urinary antigen assays during an outbreak of legionellosis in Belgium. $J$ Med Microbiol 2005; 54: 1213-6.

4. Diederen BMW, Bruin JP, Scopes E, Peeters MF, IJzerman EPF. Evaluation of the Oxoid Xpect legionella test kit for detection of Legionella pneumophila serogroup 1 antigen in urine. J Clin Microbiol 2009; 47 (7): 2272-4.

5. Guerrero C, Toldos CM, Yag?e G, et al. Comparison of diagnostic sensitivities of three assays (Bartels enzyme immunoassay - EIA, Biotest EIA, and BinaxNOW immunochromatographic test) for detection of Legionella pneumophila serogroup 1 antigen in urine. J Clin Microbiol 2004; 42: 467-8.

6. Helbig JH, Uldum SA, L?ck PC, Harrison TG. Detection of Legionella pneumophila antigen in urine samples by BinaxNOW immunochromatographic assay and comparison with both Binax Legionella urinary enzyme immunoassay (EIA) and Biotest Legionella urine antigen EIA. J Med Microbiol 2001; 50: 509-16.

7. Helbig JH, Uldum SA, Bernander S, et al. Clinical utility of urinary antigen detection for diagnosis of community-acquired, travel-associated, and nosocomial Legionnaires' disease. J Clin Microbiol 2003; 41: 838-40.

8. Rojas A, Navarro MD, Fornés FE, et al. Value of serological testing for diagnosis of legionellosis in outbreak patients. 2000; 38: 2738-9.

9. Yzerman EP, den Boer JW, Lettinga KD, et al. Sensitivity of three urinary antigen tests associated with clinical severity in a large outbreak of Legionnaires' disease in the Netherlands. J Clin Microbiol 2002; 40: 3232-6.

10. Wever PC, Yzerman EP, Kuijper EJ, Speelman P, Dankert J. Rapid diagnosis of Legionnaires' disease using an immunochromatographic assay for Legionella pneumophila serogroup 1 antigen in urine during an outbreak in the Netherlands. J Clin Microbiol 2000; 38: 2738-9. 